Supplement of Earth Surf. Dynam., 6, 451-466, 2018

https://doi.org/10.5194/esurf-6-451-2018-supplement

C Author(s) 2018. This work is distributed under

the Creative Commons Attribution 4.0 License.

(c) (1)

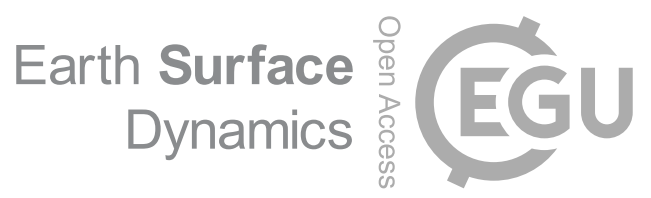

Supplement of

\title{
On the Holocene evolution of the Ayeyawady megadelta
}

Liviu Giosan et al.

Correspondence to: Liviu Giosan (lgiosan@whoi.edu)

The copyright of individual parts of the supplement might differ from the CC BY 4.0 License. 
29 Fig. S1. Trench and drill sites location and other locales mentioned in text.

30

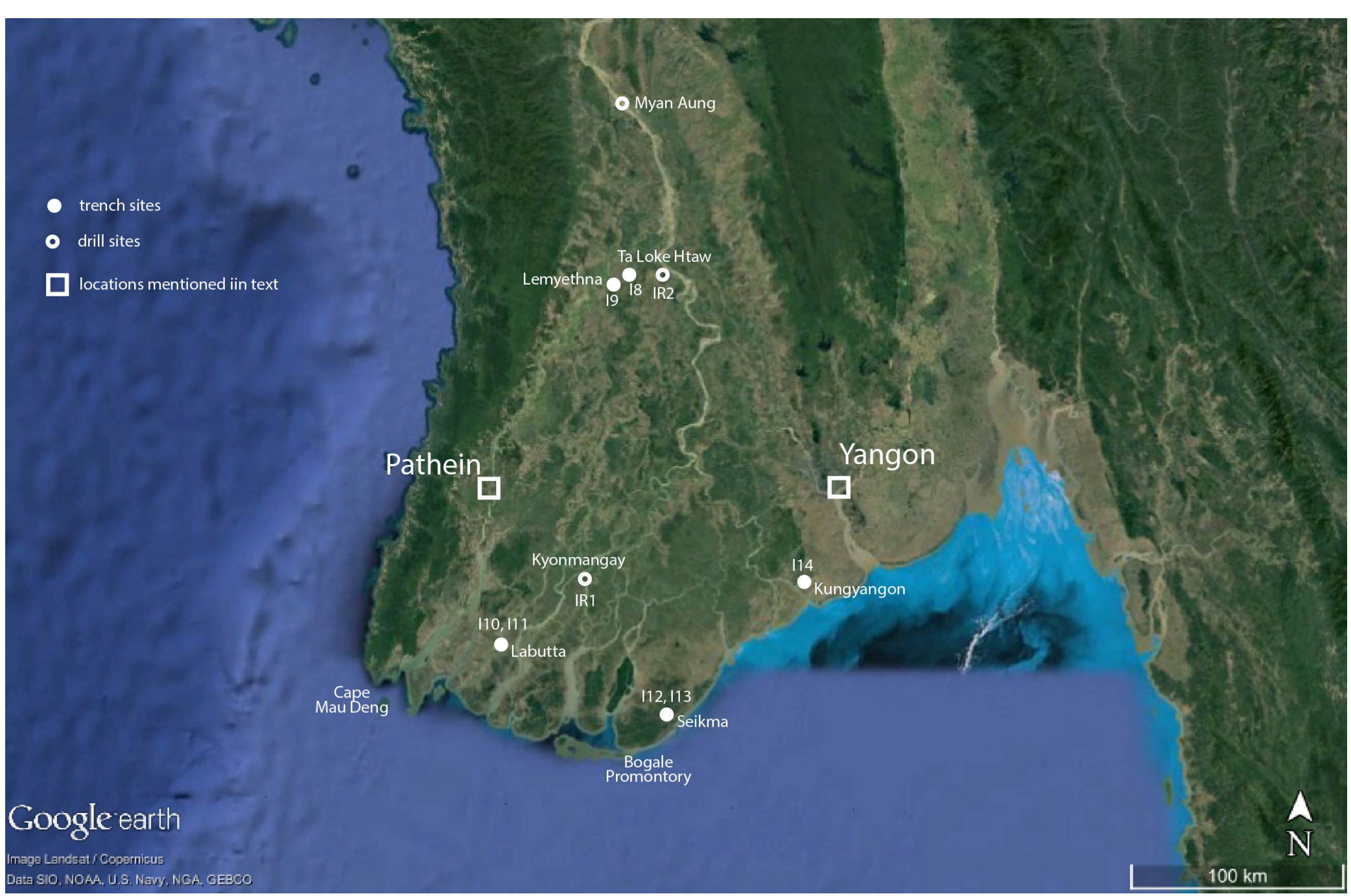


Fig. S2. Representative OSL decay curves for sample 177204 (cyan) and for standard Risø calibration quartz (black), which is known to be dominated by the fast component (Hansen, V., Murray, A.S., Buylaert, J.-P., Yeo, E.Y., Thomsen, K.J., 2015. A new irradiated quartz for beta source calibration. Radiation Measurements 81, 123-127.). Data were normalised to the initial signal intensity (first 0-0.16s).

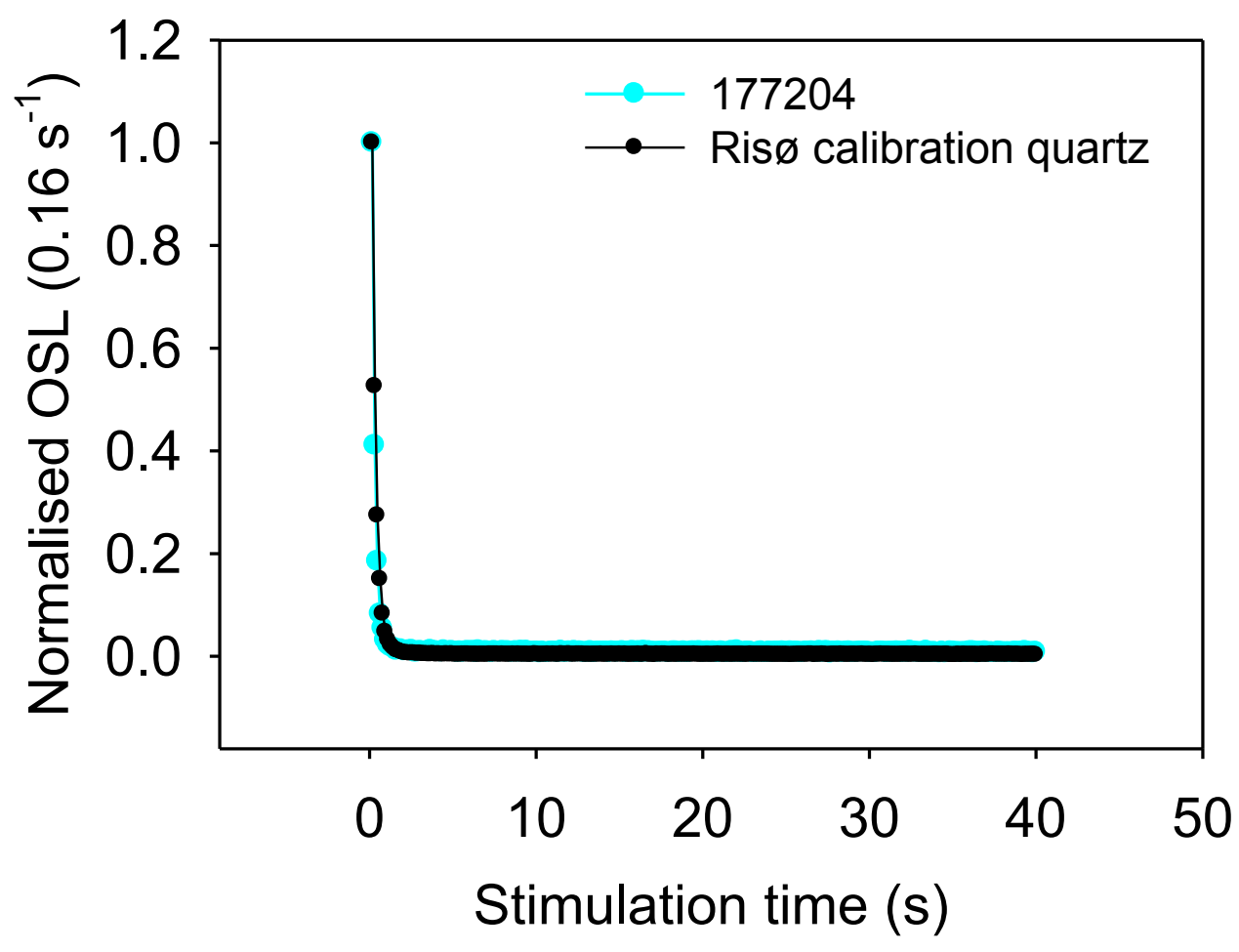


Table S1. Radionuclide activities obtained from high resolution gamma spectrometry. These values were used to derive total dose rates to quartz and K-feldspar grains presented in Table 2 using the conversion factors from Guérin et al. (2011). For K-feldspar the internal beta dose rate was estimated using an internal $\mathrm{K}$ content of $12.5 \pm 0.5 \%$ (Huntley and Baril, 1997). A cosmic ray dose rate component was also incorporated (Presscott and Hutton, 1994)

\begin{tabular}{ccccccccc} 
Sample code & $\begin{array}{c}\mathbf{U - 2 3 8} \\
\mathbf{( B q} / \mathbf{k g})\end{array}$ & $\begin{array}{c}\mathbf{R a - 2 2 6} \\
\mathbf{( B q} / \mathbf{k g})\end{array}$ & $\begin{array}{c}\mathbf{P b - 2 1 0} \\
\mathbf{( B q} / \mathbf{k g})\end{array}$ & $\begin{array}{c}\mathbf{T h}-232 \\
\mathbf{( B q} / \mathbf{k g})\end{array}$ & $\begin{array}{c}\mathbf{K}-40 \\
\mathbf{( B q} / \mathbf{k g})\end{array}$ \\
\hline 17 & 72 & 01 & $16 \pm 15$ & $27.2 \pm 1.2$ & n.a. \pm n.a. & $48.0 \pm 1.3$ & $450 \pm 22$ \\
17 & 72 & 02 & $36 \pm 5$ & $32.4 \pm 0.9$ & $39 \pm 6$ & $52.3 \pm 0.9$ & $513 \pm 14$ \\
17 & 72 & 03 & $17 \pm 4$ & $18.6 \pm 0.8$ & $17 \pm 5$ & $32.2 \pm 0.7$ & $518 \pm 13$ \\
17 & 72 & 04 & $25 \pm 3$ & $26.6 \pm 0.5$ & $27 \pm 4$ & $45.8 \pm 0.6$ & $493 \pm 10$ \\
17 & 72 & 05 & $23 \pm 3$ & $23.2 \pm 0.6$ & $25 \pm 4$ & $43.9 \pm 0.6$ & $476 \pm 10$ \\
17 & 72 & 06 & $8 \pm 4$ & $13.7 \pm 0.6$ & $15 \pm 5$ & $28.0 \pm 0.7$ & $562 \pm 14$ \\
17 & 72 & 07 & $24 \pm 4$ & $16.3 \pm 0.8$ & $14 \pm 5$ & $36.3 \pm 0.7$ & $555 \pm 14$
\end{tabular}

\section{References:}

Huntley, D. J., Baril, M. R., 1997. The K content of the K-feldspars being measured in optical dating or in thermoluminescence dating. Ancient TL 15: 11-13. Guérin G, Mercier N, Adamiec G. 2011. Dose-rate conversion factors: update. Ancient TL 29: 5-8.

Prescott JR, Hutton JT. 1994. Cosmic ray contributions to dose rates for luminescence and ESR dating: large depths and long-term time 
Table S2. Radiogenic isotope data for the Ayeyawady and nearby rivers sediments.

49

\begin{tabular}{|c|c|c|c|c|c|c|c|c|c|}
\hline Site & Preparation & Setting & Latitude & Longitude & Depth, cm & $87 \mathrm{Sr} / 86 \mathrm{Sr}$ & $43 \mathrm{Nd} / 144 \mathrm{Nd}$ & $\varepsilon N d$ & Source \\
\hline Ayeyawady (I8) & bulk sample & fluvial levee & $17^{\circ} 38^{\prime} 36.82^{\prime \prime} \mathrm{N}$ & $95^{\circ} 18^{\prime} 33.64 " \mathrm{E}$ & 95 & 0.7120 & 0.512263 & -7.3 & this study \\
\hline Ayeyawady (I12) & bulk sample & beach ridge & $15^{\circ} 50^{\prime} 10.50 " \mathrm{~N}$ & $95^{\circ} 29^{\prime} 51.00 " \mathrm{E}$ & 100 & 0.7118 & 0.512285 & -6.9 & this study \\
\hline Ayeyawady (I12) & decarbonated & beach ridge & $15^{\circ} 50^{\prime} 10.50^{\prime \prime} \mathrm{N}$ & $95^{\circ} 29^{\prime} 51.00 " \mathrm{E}$ & 100 & 0.7119 & 0.512287 & -6.8 & this study \\
\hline Ayeyawady & bulk sample & fluvial & $18^{\circ} 49^{\prime} 43.30^{\prime \prime} \mathrm{N}$ & $95^{\circ} 12^{\prime} 19.70 " \mathrm{E}$ & & & & -8.3 & Allen et al. (2008) \\
\hline Ayeyawady & bulk sample & fluvial & & & & 0.7135 & & -10.7 & Colin et al. (1999) \\
\hline Yangon River & bulk sample & fluvial & & & & 0.7080 & & -12.2 & Damodararao et al. (2016) \\
\hline Sittaung & bulk sample & fluvial levee & $17^{\circ} 27^{\prime} 9.78^{\prime \prime N}$ & $96^{\circ} 51^{\prime} 0.12 " \mathrm{E}$ & 100 & 0.7168 & 0.512105 & -10.4 & this study \\
\hline Thanlwin & bulk sample & fluvial & & & & $0.7314 \div 0.7318$ & & $-14.7 \div-15.4$ & Damodararao et al. (2016) \\
\hline
\end{tabular}

50
51 